\title{
Macula Densa
}

National Cancer Institute

\section{Source}

National Cancer Institute. Macula Densa. NCI Thesaurus. Code C33043.

An area of densely packed modified epithelial cells lining the wall of the distal convoluted tubule of the kidney that is adjacent to the afferent arteriole just before it enters the glomerulus and is in direct apposition to the juxtaglomerular cells. 